\title{
Magnetic resonance imaging features of COVID-19-related cranial nerve lesions
}

\author{
Diogo Goulart Corrêa, 1 (D) - Luiz Celso Hygino da Cruz Jr. ${ }^{1,2} \cdot$ Fernanda Cristina Rueda Lopes $^{1,2} \cdot$ Cláudio \\ de Carvalho Rangel ${ }^{1,2}$. Anna Luiza de Araújo Henriques Tessarollo ${ }^{2} \cdot$ Karla Cristina Godeiro Coelho $^{2}$. \\ Rafael Zandonadi Brandão ${ }^{2}$. Ricardo Augusto Faro Novis ${ }^{2}$. Shenia Sbardellotto Colnaghi Novis ${ }^{2}$. \\ Rodrigo Silveira ${ }^{2,3} \cdot$ Viviane T. Carvalho $^{2,3}$. Osvaldo J. M. Nascimento ${ }^{2,3}$
}

Received: 12 October 2020 / Revised: 10 November 2020 / Accepted: 8 December 2020 / Published online: 18 January 2021

(c) Journal of NeuroVirology, Inc. 2021

\begin{abstract}
The complete features of the neurological complications of coronavirus disease 2019 (COVID-19) still need to be elucidated, including associated cranial nerve involvement. In the present study we describe cranial nerve lesions seen in magnetic resonance imaging (MRI) of six cases of confirmed COVID-19, involving the olfactory bulb, optic nerve, abducens nerve, and facial nerve. Cranial nerve involvement was associated with COVID-19, but whether by direct viral invasion or autoimmunity needs to be clarified. The development of neurological symptoms after initial respiratory symptoms and the absence of the virus in the cerebrospinal fluid (CSF) suggest the possibility of autoimmunity.
\end{abstract}

Keywords SARS-CoV-2 $\cdot$ COVID-19 $\cdot$ Cranial nerves $\cdot$ MRI

\section{Introduction}

The severe acute respiratory syndrome coronavirus-2 (SARS-CoV-2) belongs to a family of single-stranded ribonucleic acid (RNA) viruses, together with the Middle East respiratory syndrome coronavirus (MERS-CoV) and the severe acute respiratory syndrome coronavirus (SARS-CoV) (Gutiérrez-Ortiz et al. 2020), which share similar structures and pathogenic mechanisms, including growing evidence of neurotropism (Wu et al. 2020).

The occurrence of neurological complications in the coronavirus disease 2019 (COVID-19), caused by SARS$\mathrm{CoV}-2$, varies according to the population and symptoms. For example, up to $36.4 \%$ of hospitalized patients in

Diogo Goulart Corrêa

diogogoulartcorrea@yahoo.com.br

1 Clínica de Diagnóstico Por Imagem (CDPI)/DASA, Avenida das Américas, 4666, 302A, 303, 307, 325, 326, Barra da Tijuca, Rio de Janeiro, RJ 2640-102, Brazil

2 NEUROCOVID-RIO Group-Research Group On Neurological Syndromes Associated With SARS-CoV-2, Rio de Janeiro, Brazil

3 Department of Neurology, Universidade Federal Fluminense, Rio de Janeiro, Brazil
Wuhan, China, presented with neurological complications (Mao et al. 2020), whereas $19.4 \%$ of patients in Italy had sensory dysfunction, and, in Germany, approximately $88.5 \%$ and $88.0 \%$ of patients reported olfactory and gustatory dysfunction, respectively (Zubair et al. 2020). Headache, dizziness, and taste and smell impairment are among the most common symptoms (Gutiérrez-Ortiz et al. 2020; Mao et al. 2020). However, the complete clinical features and pathophysiology of neurological complications of COVID19 still need to be fully elucidated. The exact nature and mechanisms of neurological manifestations, whether they occur by direct neural invasion or by an autoimmune mechanism, remain largely unknown (Gutiérrez-Ortiz et al. 2020; Khatoon et al. 2020). In this sense, several neurological complications have been reported in COVID19 and infections caused by other coronaviruses (Wu et al. 2020).

Since the beginning of the current pandemic, some authors have reported COVID-19-associated cranial nerve involvement, detected by clinical examination and/ or magnetic resonance imaging (MRI), including anosmia (with involvement of the olfactory bulb) (Aragão et al. 2020; Vargas-Gandica et al. 2020), Miller-Fischer syndrome (Lantos et al. 2020; Dinkin et al. 2020), facial paralysis (Goh et al. 2020; Lima et al. 2020), abducens nerve palsy, 
and optic nerve sheath enhancement (Dinkin et al. 2020), demonstrating the potential association between cranial nerve involvement and SARS-CoV-2. In this article, we present the MRI features of a case series with COVID-19related cranial nerve involvement.

\section{Material and methods}

The diagnosis of COVID-19 infection was based on the detection of the SARS-CoV-2 genome by reverse transcription-polymerase chain reaction (RT-PCR), from material obtained using a nasopharyngeal swab. All patients tested negative for other respiratory viruses, such as influenza, adenovirus, herpes simplex virus, varicella zoster virus, respiratory syncytial virus, and metapneumovirus. Because the patients did not have severe symptoms, only one patient (case 5) had a lumbar puncture, with negative RT-PCR analysis in the cerebrospinal fluid (CSF), due to the suspicion of Guillain-Barré syndrome.

All participants presented with the typical respiratory symptoms of COVID-19, as well as characteristic signs and symptoms of at least one cranial nerve lesion a few days after the initial symptoms of COVID-19. No participant presented with severe respiratory acute syndrome, used chloroquine or hydroxychloroquine, corticosteroids, or other drugs before the onset of the neurological symptoms.

MRI was performed in 1.5 or $3 \mathrm{~T}$ clinical scanners (Avanto, Aera, Symphony and Prisma; Siemens, Erlangen, Germany), searching for brain and cranial nerve injuries related to COVID-19, according to clinical signs and symptoms. The MRI protocol included T1-weighted imaging, with and without fat saturation, 3-dimensional Magnetization Prepared Rapid Gradient Echo (3D-MPRAGE) T1-weighted imaging, with and without intravenous injection of a gadolinium-based contrast agent, axial and coronal T2-weighted imaging, axial fluid attenuated inversion recovery (FLAIR), axial diffusionweighted imaging, axial susceptibility-weighted imaging (SWI), three-dimensional constructive interference in steady state (CISS), and short tau inversion recovery (STIR).

\section{Case series}

Case 1: A previously healthy 41-year-old woman presented with malaise and cough. The nasopharyngeal swab RTPCR was positive for SARS-CoV-2. In the second week, the patient started to present loss of sense of taste and smell. Brain MRI, performed 1 week after neurological symptoms onset, revealed bilateral gadolinium-enhancement on fat saturation T1-weighted imaging, in the olfactory bulb (Fig. 1). Two months after the neurological symptoms onset, the patient still has anosmia and ageusia; however, she did not receive a specific treatment for the neurological symptoms.

Case 2: A previously healthy 27-year-old woman presented with fever and cough. Four days after initial symptoms, the nasopharyngeal swab was positive for SARSCoV-2. Two weeks after the beginning of symptoms, she started to present blurred vision and pain in the left eye, with clinical suspicion of optic neuritis. This diagnosis was confirmed, by a MRI, performed 2 days after ocular symptoms onset, which revealed a hyperintense signal on STIR, with gadolinium enhancement, in the left optic nerve, suggestive of optic neuritis (Fig. 2). There were no other lesions in the brain parenchyma and spinal cord. Antiaquaporin-4 antibody was negative in the serum. The patient was treated with 5 days of methylprednisolone $1 \mathrm{~g} /$ day, with improvement of the symptoms.

Case 3: A 25-year-old previously healthy woman presented with mild dyspnea and fever. In the sixth day
Fig. 1 COVID-19 associated with anosmia. Fat saturation T1-weighted spin-echo MRI before $\mathbf{a}$ and after intravenous gadolinium injection $\mathbf{b}$ demonstrated bilateral enhancement in the olfactory bulb (arrows)

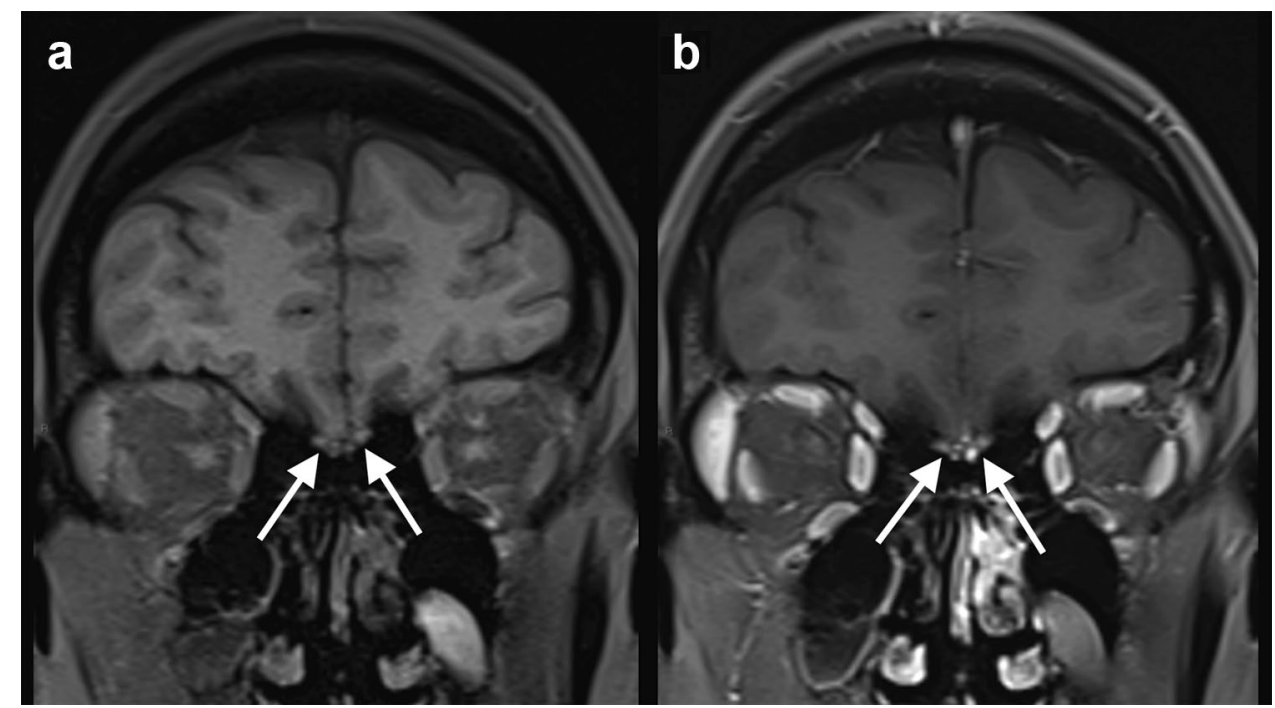


Fig. 2 COVID-19 associated with left optic neuritis. MRI showed hyperintense signal on STIR (arrow in a), as well as gadolinium enhancement in the left optic nerve (arrow in b), as seen on T1-weighted spin-echo imaging suggestive of optic neuritis. The brain parenchyma was normal. The patient did not have a history of previous demyelinating disease

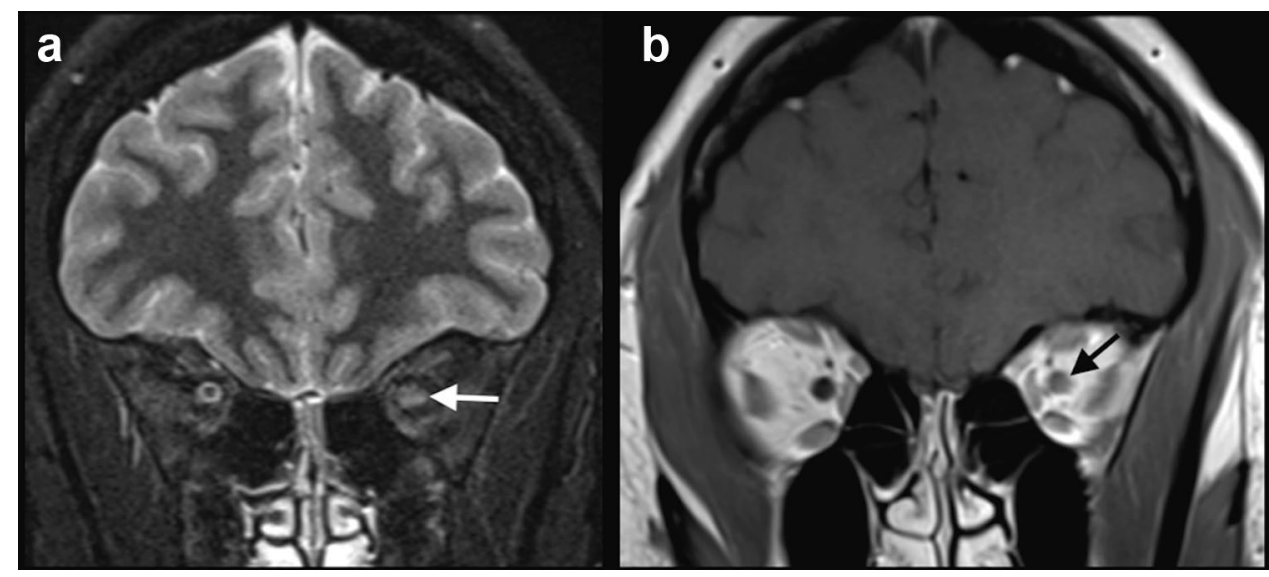

of symptoms, the nasopharyngeal swab was positive for SARS-CoV-2. After two weeks of symptoms, she presented with vertigo, muscle weakness in the right side of the face, and difficulty in closing the right eye, suggestive of right facial nerve palsy. She also presented convergent strabismus in the right eye, secondary to right abducens nerve palsy. A brain MRI was performed 3 days after the neurological symptoms onset, which demonstrated a hyperintense focus on T2-weighted imaging, associated with restricted diffusion, in the caudal portion of the pons, medial to the sulcus limitans, in the right abducens nerve nucleus. An asymmetrical gadolinium enhancement in the mastoid portion of the right facial nerve also appeared in the MRI (Fig. 3). The patient was treated with oral prednisone, $60 \mathrm{mg} / \mathrm{day}$, for 7 days, with complete recovery.

Case 4: A 30-year-old previously healthy woman presented with mild fever and sore throat. A nasopharyngeal swab was positive for SARS-CoV-2, after 5 days presenting symptoms. Ten days after the initial symptoms, she presented with right facial nerve palsy. Brain MRI, performed 4 days after, showed gadolinium enhancement in the canalicular portion of the right facial nerve and in the right geniculate ganglion (Fig. 4). The patient presented complete recovery after 5 days of treatment with oral prednisone, $60 \mathrm{mg} / \mathrm{day}$.
Fig. 3 COVID-19 associated with right facial and abducens nerve palsy. Brain MRI demonstrated a discrete hyperintense focus on $\mathrm{T} 2$-weighted imaging in the right abducens nerve nucleus (arrow in a), associated with restricted diffusion (arrow in b). MRI also revealed gadolinium enhancement in the mastoid portion of the right facial nerve, on T1-weighted spin-echo imaging (arrows in c) and on T1-3D MPRAGE (arrow in d). Although enhancement in the mastoid segment of the facial nerve can occur secondary to physiological circumneural venous plexus, this finding was asymmetrical, and correlated with the clinical symptoms

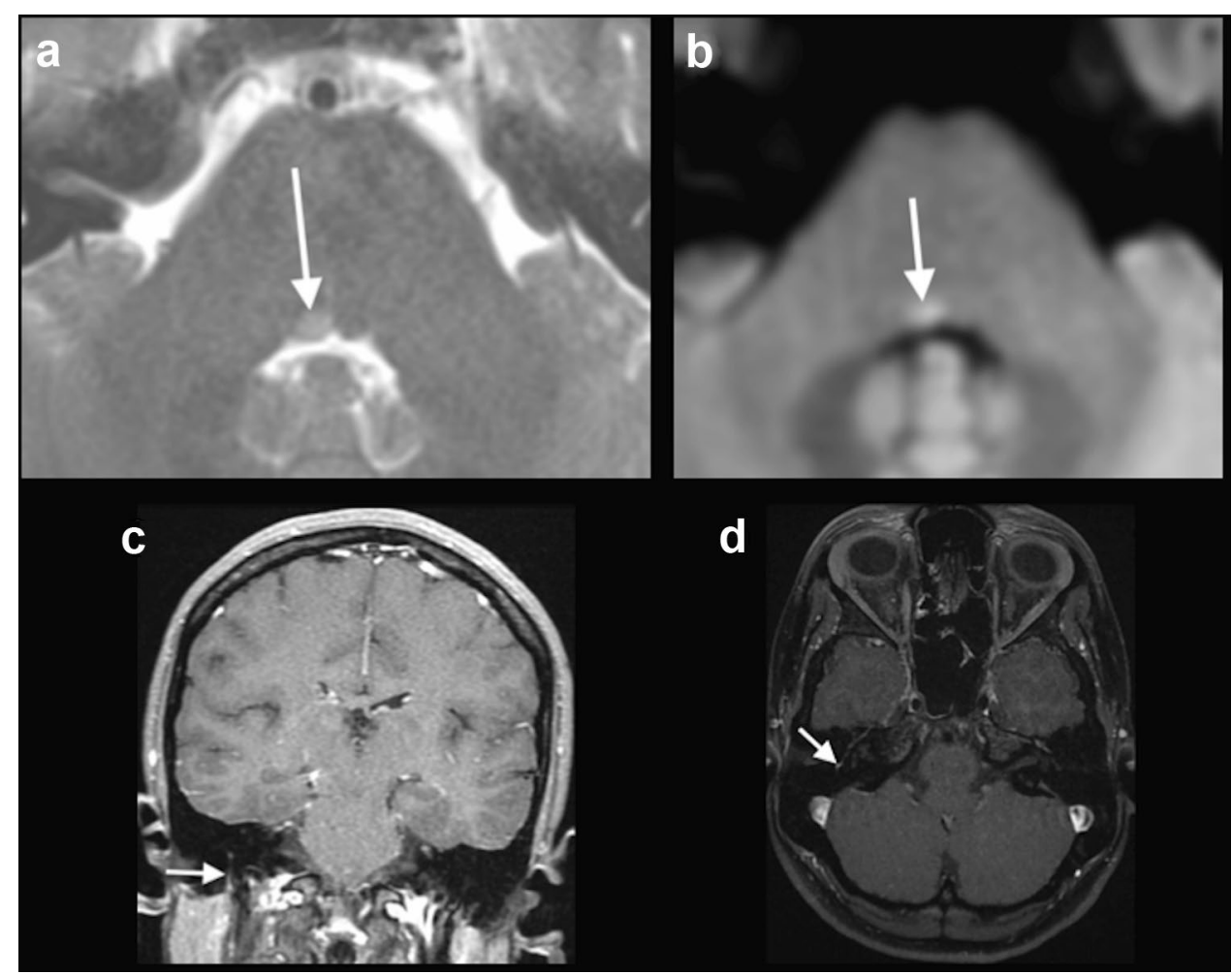


Fig. 4 COVID-19 associated with right facial nerve palsy. MRI demonstrated gadolinium enhancement in the canalicular portion of the right facial nerve (arrows) and geniculate ganglion, on T1-weighted spinecho imaging

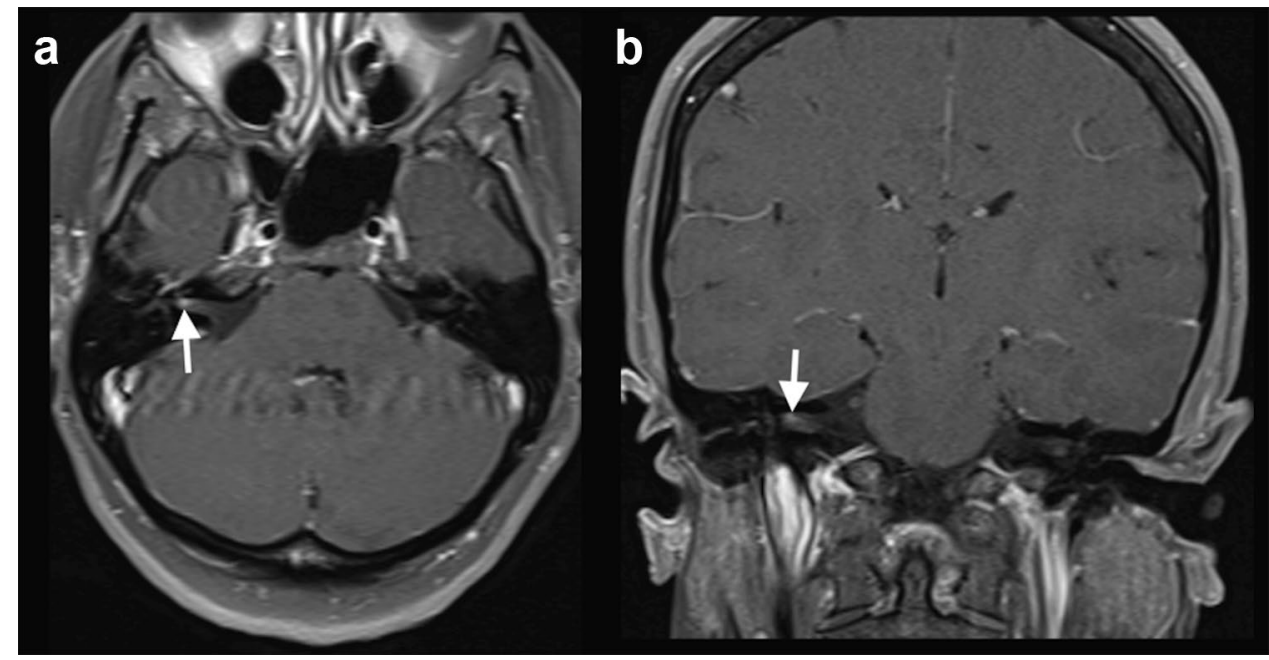

Case 5: A 65-year-old man with a history of osteoarthritis and atrial fibrillation presented with fever and generalized myalgia. A nasal swab was positive for SARS-CoV-2 during the first week of symptoms. After 2 weeks, he started having a headache and symptoms of left facial nerve palsy and lower limbs weakness. Neurologic examination revealed signs of bilateral facial nerve palsy, predominately in the left side, associated with lower limbs areflexia. A brain MRI was performed 1 week after the neurological symptoms onset, which showed bilateral gadolinium enhancement in the canalicular and labyrinthine portions of the facial nerves (Fig. 5). Lumbar spine MRI showed gadolinium enhancement in the cauda equina nerve roots. Electroneuromyography was suggestive of acute inflammatory demyelinating polyneuropathy, and CSF examination showed no cells, protein level: $100 \mathrm{mg} / \mathrm{dl}$, compatible with GuillainBarré syndrome. RT-PCR for SARS-CoV-2 in the CSF was negative. The patient received intravenous immunoglobulin, and presented partial improvement of the neurological symptoms.

Case 6: A 33-year-old previously healthy man presented with fever for one week, associated with a positive nasal swab for SARS-CoV-2. After 2 weeks, he started with bilateral facial nerve palsy. Brain MRI, performed 3 days after the neurological symptoms onset, demonstrated gadolinium enhancement in the canalicular and labyrinthine portions of the facial nerves (Fig. 6). The patient was also treated with oral prednisone, $60 \mathrm{mg} /$ day, for 7 days, with complete recovery.

In all the six cases, brain MRI did not reveal additional brain parenchymal lesions.
Fig. 5 COVID-19 associated with bilateral facial nerve palsy. Brain MRI showed bilateral gadolinium enhancement in the canalicular and labyrinthine portions of the facial nerves on axial T1-weighted spin-echo imaging (arrows in a) and coronal fat saturation T1-weighted spin-echo imaging (arrows in b)

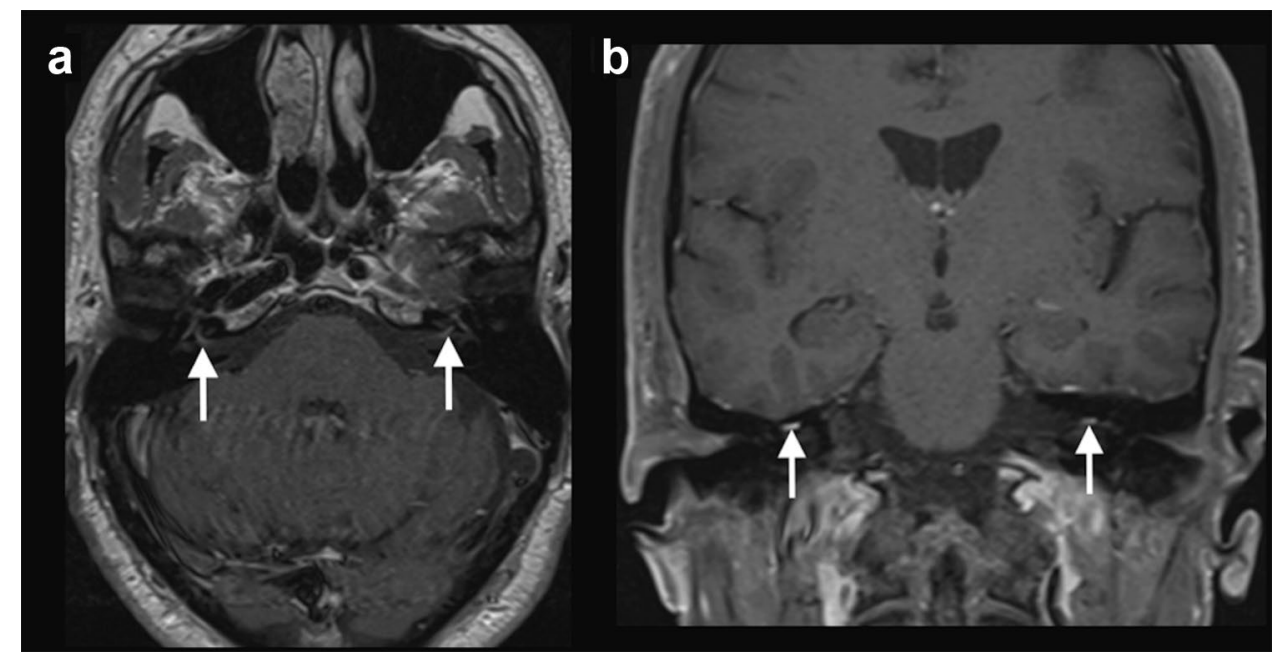


Fig. 6 COVID-19 associated with bilateral facial nerve palsy. Brain MRI showed bilateral gadolinium enhancement in the canalicular and labyrinthine portions of the facial nerves, on T1-3D MPRAGE (arrow in a) and coronal fat saturation T1-weighted spin-echo imaging (arrow in b)

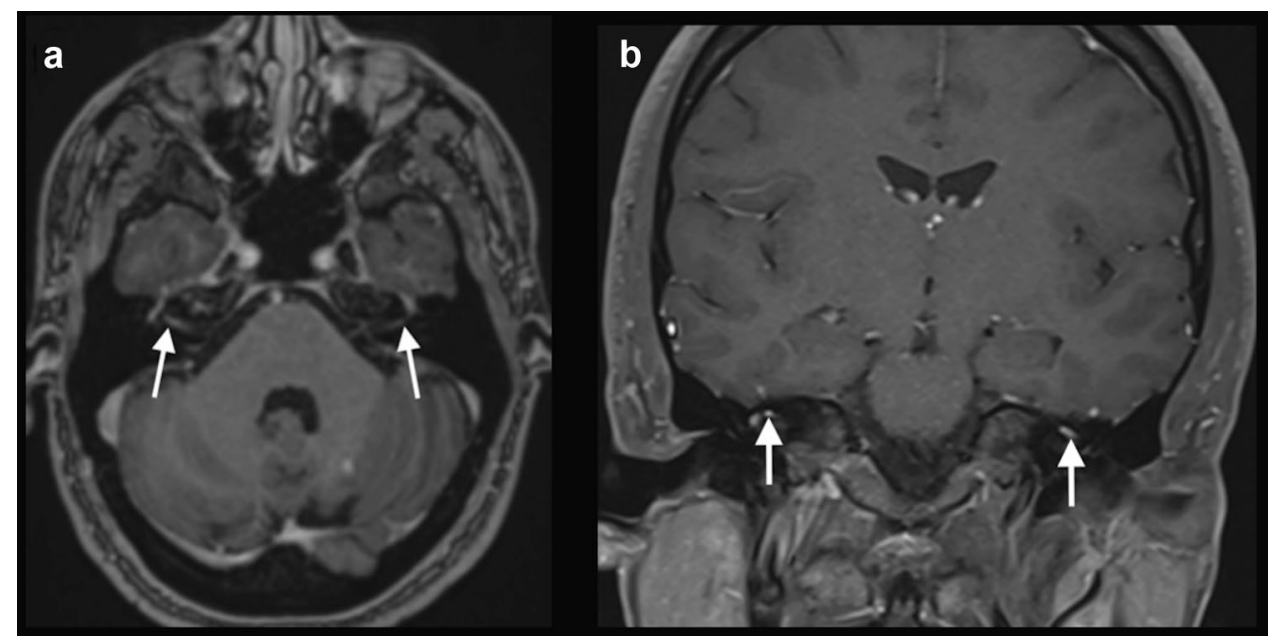

\section{Discussion}

We reported cranial nerve abnormalities as seen by MRI of six confirmed cases of COVID-19. One participant had bilateral gadolinium-enhancement in the olfactory bulb, associated with anosmia; one patient had left optic neuritis; one case presented with a lesion in the right abducens nerve nucleus; two participants had facial palsy, one unilaterally, and the other bilaterally; and one case had bilateral facial palsy associated with the Guillain-Barré syndrome.

Although COVID-19 can have a series of neurological complications, it is still unclear if SARS-CoV-2 is neurotropic in humans. However, there are some plausible hypotheses for viral neural entry, such as via the olfactory bulb, through the vascular endothelium, by leukocyte migration across the blood-brain barrier, and by transsynaptic transfer across infected neurons (Zubair et al. 2020). It has been proposed that SARS$\mathrm{CoV}-2$ binds by spike proteins expressed on its surface to the angiotensin-converting enzyme 2 (ACE2) to enter into the host's cells (Hoffmann et al. 2020). ACE2 is expressed in the airway epithelium, kidney cells, small intestine, lung parenchyma, vascular endothelium, and widely throughout the CNS, including the substantia nigra, ventricles, middle temporal gyrus, posterior cingulate cortex, and olfactory bulb (Zubair et al. 2020). Several other viruses can use the olfactory nerve as a shortcut into the CNS, such as influenza A virus, herpesvirus, poliovirus, paramyxovirus, rabies virus, parainfluenza virus, adenovirus, Japanese encephalitis virus, West Nile virus, and chikungunya virus, among others (van Riel et al. 2015).

Previous studies have demonstrated imaging alterations in the olfactory bulb and/or adjacent gyrus rectus, secondary to COVID-19, in patients with anosmia, using T1-weighted imaging with fat suppression, pregadolinium and postgadolinium, and FLAIR, corroborating that the olfactory bulb is a potential entry site into the CNS for SARS-CoV-2 (Aragão et al. 2020; Politi et al. 2020; Vargas-Gandica et al. 2020; Klironomos et al. 2020). Similarly, in our case series, one patient presented with MRI-detected alterations in the olfactory bulb, seen in fat saturation post-gadolinium T1-weighted imaging.

We also presented five cases of confirmed COVID-19 with lesions in other cranial nerves that did not present associated anosmia, suggesting that the neuronal injury in this disease goes beyond the invasion of the olfactory bulb. Previous authors reported COVID-19-related facial palsy (Lima et al. 2020; Klironomos et al. 2020). Similarly, we reported one patient with unilateral facial nerve palsy. In addition, we also reported two cases with bilateral facial nerve palsy. Facial nerve palsy, unilateral or bilateral, is associated with several other infections, such as by herpes simplex virus, varicella zoster, human immunodeficiency virus, influenza virus, poliomyelitis virus, human T-cell lymphotrophic virus, mycobacterium tuberculosis, infectious mononucleosis, Lyme disease, and syphilis, as well as with non-infectious causes, including sarcoidosis and neoplasms (Goh et al. 2020; Jain et al. 2006). The exact pathogenesis of acute facial nerve palsy remains unclear in COVID-19, but in association with neurotropic viruses, one can hypothesize that it is related with axonal spread and viral replication leading to inflammation and demyelination (Goh et al. 2020; Lima et al. 2020). However, previous authors have failed to detect SARS-CoV-2 in the CSF, so cranial neuropathies are probably related to immune-mediated injury from proinflammatory cytokines, with or without molecular mimicry (Fotuhia et al. 2020), rather than direct viral neurotropism (Gutiérrez-Ortiz et al. 2020). In addition, it is important to remember that not all facial nerve 
enhancement is pathological. Mild enhancement in the geniculate ganglion, tympanic, or mastoid segments can be normal, presumably secondary to the circumneural venous plexus in these segments. However, when asymmetrical, correlated with symptoms, associated with other facial nerve segments enhancement, or with other abnormalities, such as facial canal expansion, it can be considered pathological (Raghavan et al. 2009). Brain MRI of our case 3 demonstrated gadolinium-enhancement in the mastoid portion of the right facial nerve. Although this finding could be related to the circumneural facial venous plexus, as it correlated with clinical symptoms, and it was asymmetric in relation to the left side, we could consider it as a pathological finding.

Abducens nerve palsy has also been previously reported, in the context of Miller Fisher syndrome, but without imaging evidence of nerve involvement (Dinkin et al. 2020; Gutiérrez-Ortiz et al. 2020). We presented a case with involvement of the abducens nucleus in the pons associated with facial palsy, but not related to the Miller Fischer syndrome, since the patient did not preset ataxia or hyporeflexia.

Several studies have associated COVID-19 with GuillainBarré syndrome with axonal neuropathy, demyelination, or cranial nerve lesions, which probably occurs due to molecular mimicry (Fotuhia et al. 2020; Toscano et al. 2020). In the present study, we reported one case of Guillain-Barré syndrome with bilateral facial palsy by MRI. Guillain-Barré syndrome diagnosis is confirmed by clinical parameters, CSF study, and electroneuromyography. MRI can be performed to exclude other causes of flaccid paralysis and show contrast enhancement in the cauda equina nerve roots. However, nerve root enhancement can be seen in several causes of radiculitis and is not pathognomonic.

Dinkin et al. (2020) presented a COVID-19 case with contrast enhancement in the optic nerve perineural sheath without involvement of the nerve substance. The authors interpreted this finding as a possible viral leptomeningeal invasion, though nonspecific. Other authors have reported prominent subarachnoid spaces around the optic nerves, probably due to increased intracranial pressure in COVID-19 (Klironomos et al. 2020). On the other hand, we presented a case with hyperintense signal in the optic nerve substance associated with contrast enhancement. Coronaviruses can cause clinical entities such as conjunctivitis, anterior uveitis, retinitis, and optic neuritis in feline and murine models (Seah et al. 2020). Previous case reports have described CNS demyelinating lesions induced by a delayed immune response secondary to viremia, complicating COVID-19 (Zanin et al. 2020; Corrêa et al. 2020). Also, experimental models using human coronavirus have indicated a potential link between coronavirus infection and the development of demyelinating diseases (Montalvan et al. 2020). Although there is only sparse evidence, optic neuritis may also be considered a demyelinating lesion, and may be induced by a delayed immune response.

Although cranial nerve lesions can complicate COVID19 , this association has been reported only in case reports or short case series, as in the present study. Therefore, the real prevalence of this type of neurological complication is not known. A limitation of this work is that we did not perform lumbar punctures on all of our patients. However, since all participants had mild and self-limiting respiratory symptoms and neurological complications appeared after improvement of the initial symptoms, there was a marked temporal relationship with the SARS-CoV-2 infection. Also, all participants had their SARS-CoV-2 infection confirmed by RT-PCR and tested negative for other respiratory viruses.

In conclusion, although the full neurological features of SARS-CoV-2 infection are still to be characterized, cranial nerve lesions are part of this spectrum, and MRI can help to detect these abnormalities. Further research is still needed to describe the incidence of cranial nerve lesions in COVID-19.

\section{Compliance with ethical standards}

Conflict of interest The authors declare that they have no conflict of interest.

\section{References}

Aragão MFVV, Leal MC, Cartaxo Filho OQ, Fonseca TM, Valença MM (2020) Anosmia in COVID-19 associated with injury to the olfactory bulbs evident on MRI. AJNR Am J Neuroradiol 41:1703-1706

Corrêa DG, Lima FCS, Bezerra DC, Coutinho Júnior AC, Hygino da Cruz Júnior LC (2020) COVID-19 associated with encephalomyeloradiculitis and positive antiaquaporin- 4 antibodies: cause or coincidence? Mult Scler [Online ahead of print].

Dinkin M, Gao V, Kahan J et al (2020) COVID-19 presenting with ophthalmoparesis from cranial nerve palsy. Neurology 95:221-223

Fotuhia M, Mianc A, Meysamid S, Rajic CA (2020) Neurobiology of COVID-19. Am J Alzheimers Dis 76:3-19

Goh Y, Beh DLL, Makmur A, Somani J, Chan ACY (2020) Pearls and oysters: facial nerve palsy as a neurological manifestation of Covid-19 infection. Neurology 95:364-367

Gutiérrez-Ortiz C, Méndez A, Rodrigo-Rey S et al (2020) Miller Fisher syndrome and polyneuritis cranialis in COVID-19. Neurology 95:e601-e605

Hoffmann M, Kleine-Weber H, Schroeder S et al (2020) SARS-CoV-2 cell entry depends on ACE2 and TMPRSS2 and is blocked by a clinically proven protease inhibitor. Cell 181:271-280

Jain V, Deshmukh A, Gollomp S (2006) Bilateral facial paralysis case presentation and discussion of differential diagnosis. J Gen Intern Med 21:C7-C10

Khatoon F, Prasad K, Kumar V (2020) Neurological manifestations of COVID-19: available evidences and a new paradigm. J Neurovirol 26:619-630 
Klironomos S, Tzortzakakis A, Kits A, et al (2020) Nervous system involvement in COVID-19: results from a retrospective consecutive neuroimaging cohort. Radiology [Online ahead of print].

Lantos JE, Strauss SB, Lin E (2020) COVID-19- associated miller fisher syndrome: MRI findings. AJNR Am J Neuroradiol 41:1184-1186

Lima MA, Silva MTT, Soares CN, et al (2020) Peripheral facial nerve palsy associated with COVID-19. J Neurovirol [Online ahead of print].

Mao L, Jin H, Wang M et al (2020) Neurologic manifestations of hospitalized patients with coronavirus disease 2019 in Wuhan, China. JAMA Neurol 77:683-690

Montalvan V, Lee J, Bueso T, De Toledo J, Rivas K (2020) Neurological manifestations of COVID-19 and other coronavirus infections: a systematic review. Clin Neurol Neurosurg 194:105921

Politi LS, Salsano E, Grimaldi M (2020) Magnetic resonance imaging alteration of the brain in a patient with coronavirus disease 2019 (COVID19) and anosmia. JAMA Neurol 77:1028-1029

Raghavan P, Mukherjee S, Phillips CD (2009) Imaging of the facial nerve. Neuroimag Clin N Am 19:407-425

Seah I, Agrawal R (2020) Can the coronavirus disease 2019 (COVID19) affect the eyes? A review of coronaviruses and ocular implications in humans and animals. Ocul Immunol Inflamm 28:391-395
Toscano G, Palmerini F, Ravaglia S et al (2020) Guillain-Barré syndrome associated with SARS-CoV-2. N Engl J Med 382:2574-2576

van Riel D, Verdijk R, Kuiken T (2015) The olfactory nerve: a shortcut for influenza and other viral diseases into the central nervous system. J Pathol 235:277-287

Vargas-Gandica J, Winter D, Schnippe R, et al (2020) Ageusia and anosmia, a common sign of COVID-19? A case series from four countries. J Neurovirol [Online ahead of print]

Wu Y, Xuc X, Chenb Z et al (2020) Nervous system involvement after infection with COVID-19 and other coronaviruses. Brain Behav Immun 87:18-22

Zanin L, Saraceno G, Panciani PP et al (2020) SARS-CoV-2 can induce brain and spine demyelinating lesions. Acta Neurochir (Wien) 162:1491-1494

Zubair AS, McAlpine LS, Gardin T, Farhadian S, Kuruvilla DE, Spudich S (2020) Neuropathogenesis and neurologic manifestations of the coronaviruses in the age of coronavirus disease 2019: a review. JAMA Neurol 77:1018-1027

Publisher's Note Springer Nature remains neutral with regard to jurisdictional claims in published maps and institutional affiliations. 